\title{
LA PARTICIPATION DES TRAVAILLEURS DANS LA DOCTRINE SOCIALE DE L'EGLISE
}

\author{
Nicolas AuberT ${ }^{1}$
}

\section{Résumé :}

La doctrine sociale de l'Eglise (DSE) fait référence aux trois formes de participation des travailleurs. La participation à la propriété de l'entreprise prend la forme de la détention de parts sociales ou d'actions de l'entreprise par les salariés. La participation à la gestion de l'entreprise se traduit d'une part par l'information et la consultation des salariés par l'entremise des institutions représentatives du personnel et d'autre part par la présence de salariés dans les instances de gouvernement de l'entreprise. Enfin, les salariés peuvent participer aux profits de l'entreprise grâce à un accord de participation (obligatoire en France pour les entreprises de plus de 50 salariés). En France, les systèmes de participation sont le fruit de plusieurs influences dont celle qu'a eu l'enseignement social de l'Eglise sur Charles de Gaulle qui fut à l'origine de l'essentiel des systèmes de participation encore en vigueur en France. La doctrine sociale de l'Eglise fait de la participation un de ses principes. Au travers des textes des encycliques sociales, nous constatons que la DSE promeut la participation pour atténuer le conflit entre le «monde du capital » et le «monde du travail » et édifier une communauté, pour favoriser une juste répartition des richesses et la prospérité.

La formule de Pie XI résume la doctrine sociale chrétienne en matière de participation : « les ouvriers et les employés sont appelés à participer en quelque manière à la propriété de l'entreprise, à sa gestion ou aux profits qu'elle apporte $»^{2}$. Le pape fait ici référence aux trois formes de participation des travailleurs. En France, plusieurs dispositions prévoient la mise en œuvre de ces formes de participation. La participation à la propriété de l'entreprise prend la forme de la détention de parts sociales ou d'actions de l'entreprise par les salariés. On parle le plus souvent dans ce cas d'actionnariat salarié. La participation à la gestion de l'entreprise se traduit d'une part par l'information et la consultation des salariés par l'entremise des institutions représentatives du personnel et d'autre part par la présence de salariés dans les instances de gouvernement de l'entreprise telles que le conseil d'administration ou le conseil de surveillance. Enfin, les salariés peuvent participer aux profits de l'entreprise grâce à un accord de participation (obligatoire en France pour les entreprises de plus de 50 salariés). De ces trois formes de participation, la participation à la gestion est la moins bien circonscrite. Elle trouve en effet des expressions très différentes selon les pratiques de management

\footnotetext{
${ }^{1}$ Aix Marseille Univ, CERGAM, IAE Aix-Marseille, Aix-en-Provence, France.

2 Pie XI (1931), Quadragesimo anno, https://www.doctrine-sociale-catholique.fr/les-textes-officiels/196quadragesimo-anno, $\$ 72$.
} 
adoptées, les différentes structures juridiques des entreprises, leur secteur, leur taille, etc. Les réalités de la société coopérative de production (SCOP) et de l'entreprise multinationale cotée sont différentes. De même, les pratiques de management participatif sont nombreuses et s'étendent des journaux d'entreprise à l'entreprise autogérée en passant par les cercles de qualité. On trouve également ces trois formes de participation regroupées en deux familles : la participation financière des salariés; - la participation des salariés aux décisions. Cette seconde typologie adoptée notamment par les anglo-saxons ${ }^{3}$ regroupe la participation à la propriété et la participation aux profits d'une part et la participation à la gestion d'autre part.

Les systèmes de participation sont le fruit de plusieurs influences. Mais une source d'inspiration majeure en France est l'enseignement social de l'Eglise depuis l'encyclique Rerum Novarum. Dès le XIX ${ }^{\text {ème }}$ siècle, des entrepreneurs chrétiens comme Léon Harmel mettent en œuvre des expériences participatives en se réclamant de la pensée chrétienne. La pensée sociale chrétienne inspirera également Charles de Gaulle qui fut à l'origine de l'essentiel des systèmes de participation encore en vigueur en France.

La doctrine sociale de l'Eglise fait de la participation un de ses principes ${ }^{4}$. Elle est une conséquence de la subsidiarité qui ne se limite pas seulement à la vie en entreprise sur laquelle nous nous focalisons. «[La participation] s'exprime, essentiellement en une série d'activités à travers lesquelles le citoyen, comme individu ou en association avec d'autres, directement ou au moyen de ses représentants, contribue à la vie culturelle, économique, sociale et politique de la communauté civile à laquelle il appartient. La participation est un devoir que tous doivent consciemment exercer, d'une manière responsable en vue du bien commun $»^{5}$. Sur la question de la participation des travailleurs à la gouvernance des entreprises, Vatican II précise après avoir insisté sur la promotion de « la participation active

\footnotetext{
${ }^{3}$ Kruse, Blasi et Freeman parlent notamment de shared capitalism et de shared decision making (Kruse, D., Freeman, R. B., \& Blasi, J. R. (Eds.). (2010). Shared capitalism at work : employee ownership, profit and gain sharing, and broad-based stock options. Chicago: The University of Chicago Press.)

${ }^{4}$ Conseil pontifical Justice et Paix, Compendium de la doctrine sociale de l'Eglise, Bayard, $\S 160$.

${ }^{5}$ Ibid $\S 184$.
} 
de tous à la gestion des entreprises » : «Et comme bien souvent ce n'est déjà plus au niveau de l'entreprise, mais à des instances supérieures, que se prennent les décisions économiques et sociales dont dépend l'avenir des travailleurs et de leurs enfants, ceux-ci doivent également participer à ces décisions, soit par eux-mêmes, soit par leurs représentants librement choisis $»^{6}$. La participation trouve particulièrement sa place dans l'entreprise puisque le Compendium énonce également que : «le rapport entre travail et capital trouve aussi une expression à travers la participation des travailleurs à la propriété, à sa gestion et à ses fruits $»^{7}$. Nous retrouvons les trois formes de participation évoquées plus haut.

Bien qu'elle soit évoquée dans de nombreux textes, on trouve plus particulièrement traitée la question de la participation dans cinq encycliques sociales : Rerum Novarum ${ }^{8}$, Quadragesimo Anno $^{9}$, Mater et Magistra ${ }^{10}$, Laborem Exercens ${ }^{11}$ et Centesimus Annus ${ }^{12}$. Dans la suite du texte, nous rappelons uniquement l'acronyme désignant les textes de la façon suivante : Rerum Novarum (RN), Quadragesimo Anno (QA), Mater et Magistra (MM), Laborem Exercens (LE), Centesimus Annus (CA) et Gaudium et Spes (GS). Nous indiquons également entre parenthèses la référence du paragraphe. On retrouve également des développements importants au sujet de la participation dans la constitution Gaudium et Spes du concile Vatican $\mathrm{II}^{13}$. Nous reportons les liens vers les textes des encycliques et du concile figurant sur le site du Vatican et, à défaut, ceux apparaissant sur le site du CERAS (centre d'études et de recherches en action sociale).

\footnotetext{
${ }^{6}$ Concile Vatican II (1965), Constitution Gaudium et Spes sur l'Eglise dans le monde de ce temps, http://www.vatican.va/archive/hist_councils/ii_vatican_council/documents/vat-ii_cons_19651207_gaudium-etspes fr.html, $\$ 68$.

${ }^{7}$ Conseil pontifical Justice et Paix, op. cit. $\$ 281$.

8 Léon XIII (1891) Rerum Novarum, http://w2.vatican.va/content/leo-xiii/fr/encyclicals/documents/hf 1xiii_enc 15051891_rerum-novarum.html.

9 Pie XI (1931), Quadragesimo anno, http://w2.vatican.va/content/pius-xi/en/encyclicals/documents/hf_pxi_enc 19310515 quadragesimo-anno.html.

${ }_{10}$ Jean XXIII (1961), Mater et magistra, http://w2.vatican.va/content/john-xxiii/fr/encyclicals/documents/hf jxxiii enc 15051961 mater.html.

${ }_{11}$ Jean-Paul II (1981), Laborem exercens, https://www.doctrine-sociale-catholique.fr/les-textes-officiels/209laborem-exercens.

12 Jean-Paul II (1991), Centesimus annus, https://www.doctrine-sociale-catholique.fr/les-textes-officiels/190centesimus-annus.

${ }^{13}$ Concile Vatican II (1965), op. cit.
} 


\section{La participation pour atténuer le conflit entre le « monde du capital » et le « monde du travail » et édifier une communauté}

Pie XI, faisant référence à la notion de juste salaire énoncée par son prédécesseur Léon XIII « condamnait la présomption de ceux qui soutiennent qu'on résout sans peine cette question très délicate (celle de l'opposition du capital et du travail) à l'aide d'une formule ou d'une règle unique, d'ailleurs absolument fausse $»^{14}$. Si les encycliques sociales ne proposent pas de modèle ${ }^{15}$, elle avance néanmoins que la participation des travailleurs dans l'entreprise puisse être une solution pour remédier aux conflits entre capital.

L'Eglise défend une vision organique de la société qui condamne l'opposition entre capital et travail ${ }^{16}$. Cette condamnation est présente dès l'encyclique Rerum Novarum de Léon XIII : « Il ne peut y avoir de capital sans travail ni de travail sans capital $»^{17}$. Le constat de cette opposition est même une des motivations de la première encyclique sociale dont le texte commence par le constat d'une modification des rapports entre « patrons et ouvriers ${ }^{18}$. JeanPaul II va jusqu'à affirmer qu'un système de travail juste dépasse l'antinomie entre travail et capital $^{19}$. La doctrine sociale de l'Eglise établit la primauté du travail sur le capital : « Il faut répéter encore une fois le principe fondamental : la hiérarchie des valeurs, le sens profond du travail exigent que le capital soit au service du travail et non le travail au service du

\footnotetext{
${ }^{14} \mathrm{QA}, \S 73$.

${ }^{15} \mathrm{CA}, \S 43$.

${ }^{16}$ Cette thèse est notamment défendue par Laurent, B. (2007). L'enseignement social de l'Eglise et l'économie de marché. Paris: Parole et Silence.

${ }^{17} \mathrm{RN}, \S 15$.

${ }^{18} \mathrm{RN}, \S 1$.

${ }^{19} \mathrm{LE}, \S 13$.
} 
capital $»^{20}$. Cette primauté du travail découle de la priorité de l'homme sur les choses ${ }^{21}$ et de l'idée que le capital est lui-même le fruit du travail des générations passées ${ }^{22}$.

Les encycliques sociales ont dénoncé les dérives du communisme et du capitalisme. Jean-Paul II dénonce les égarements des deux expériences en matière de consommation et de travail : « [La consommation] se retrouve aussi dans le travail, lorsqu'il est organisé de manière à ne valoriser que ses productions et ses revenus sans se soucier de savoir si le travailleur, par son travail, s'épanouit plus ou moins en son humanité, selon qu'augmente l'intensité de sa participation à une véritable communauté solidaire (nous soulignons), ou bien que s'aggrave son isolement au sein d'un ensemble de relations caractérisé par une compétition exaspérée et des exclusions réciproques, où il n'est considéré que comme un moyen, et non comme une fin. $»^{23}$.

En effet, selon la doctrine sociale de l'Eglise «la caractéristique du travail est avant tout d'unir les hommes et c'est en cela que consiste sa force sociale : la force de construire une communauté. En définitive, dans cette communauté, doivent s'unir de quelque manière et les travailleurs et ceux qui disposent des moyens de production ou en sont propriétaires $»^{24}$. Par cette analyse, l'encyclique du travail assimile l'entreprise à une communauté au sein de laquelle sont non seulement liés les intérêts des travailleurs entre eux mais également les intérêts des travailleurs et des propriétaires des moyens de production. On voit réapparaitre l'assimilation de l'entreprise à une communauté dans un autre texte de Jean-Paul II. Elle y devient un objectif de l'entreprise à côté de la recherche du profit: «En effet, le but de l'entreprise n'est pas uniquement la production du profit, mais l'existence même de l'entreprise comme communauté de personnes qui, de différentes manières, recherchent la

\footnotetext{
${ }^{20} \mathrm{LE}, \S 23$.

${ }^{21} \mathrm{LE}, \S 12$.

${ }^{22} \mathrm{LE}, \$ 13$.

${ }^{23} \mathrm{CA}, \S 41$.

${ }^{24} \mathrm{LE}, \S 20$.
} 
satisfaction de leurs besoins fondamentaux et qui constituent un groupe particulier au service de la société tout entière $»^{25}$.

Le pape considère que le problème du travail doit être analysé à la lumière $d u$ «grand conflit » opposant le « monde du travail » au « monde du capital ». Il déplore que, durant cette période de conflit qui n'est pas terminée, aient été opposés les détenteurs des moyens de production à « la multitude plus large des gens qui, privés de ces moyens [de production], ne participaient au processus de production que par leur travail ${ }^{26}$. L'encyclique suggère ici que, si le travail permet d'unir les hommes en une communauté, le seul travail est insuffisant pour participer pleinement au processus de production. A la «primauté absolue du capital», l'Eglise refuse d'opposer le système socialiste, et lui oppose « une société du travail libre, de l'entreprise et de la participation ${ }^{27}$. Quelles sont les modalités de cette formule proposée par l'Eglise ? Plutôt qu'elle ne propose des solutions, les encycliques valident a posteriori des expériences. Certaines de ces expériences ont toutefois été suscitées par les premières encycliques sociales. «Diverses formes de néocapitalisme ou de collectivisme se sont développées. Il n'est pas rare que les travailleurs puissent participer, et qu'ils participent effectivement, à la gestion et au contrôle de la productivité des entreprises. Au moyen d'associations appropriées, ils ont une influence sur les conditions de travail et de rémunération, comme aussi sur la législation sociale $»^{28}$. Le rôle des syndicats dans la négociation et l'atténuation des conflits entre le capital et le travail est également souligné. «Là encore, il convient de rappeler le rôle des syndicats, non seulement comme instruments de négociation mais encore comme « lieux » d'expression de la personnalité : ils sont utiles au

\footnotetext{
${ }^{25} \mathrm{CA}, \S 35$.

${ }^{26} \mathrm{LE}, \S 11$.

${ }^{27} \mathrm{CA}, \S 35$.

${ }^{28} \mathrm{LE}, \S 8$.
} 
développement d'une authentique culture du travail et ils aident les travailleurs à participer d'une façon pleinement humaine à la vie de l'entreprise $»^{29}$.

\section{La participation pour une juste répartition des richesses}

Outre les conflits sociaux qui trouvent leur source dans l'entreprise, une autre préoccupation de la première encyclique sociale que l'on retrouve jusqu'à l'encyclique de Benoît XVI Caritas in Veritate ${ }^{30}$ est celle de la juste répartition des richesses : « Mais c'est aussi un fait qu'en plusieurs de ces pays, face à la misère extrême de la multitude, s'étalent au grand jour, insultant au sort des pauvres, le luxe et les dépenses somptuaires d'une poignée de privilégiés $\rangle^{31}$. A cette répartition inégale des ressources, l’Eglise oppose le principe de la destination universelle ${ }^{32}$.

Les encycliques sociales énoncent d'abord la notion de juste salaire pour pallier les inégalités de dotation. Mais l'Eglise s'intéresse également à la répartition des richesses. Le constat de Pie XI est éloquent pour témoigner de la préoccupation de l'Eglise à propos des inégalités de richesse et de la nécessité d'y subvenir par leur meilleure répartition : «Il n’en reste pas moins vrai que l'existence d'une immense multitude de prolétaires d'une part, et d'un petit nombre de riches pourvus d'énormes ressources d'autre part, atteste à l'évidence que les richesses créées en si grande abondance à notre époque d'industrialisme sont mal réparties et ne sont pas appliquées comme il conviendrait aux besoins des différentes classes $»^{33}$.

Pie XI propose d'assurer le «relèvement du prolétariat par l'accession à la propriété ». Il appelle à «tout mettre en œuvre afin que, dans l'avenir du moins, la part des biens qui

\footnotetext{
${ }^{29} \mathrm{CA}, \S 15$.

${ }^{30}$ Benoît XVI (2009), Caritas in veritate, http://w2.vatican.va/content/benedict-

xvi/fr/encyclicals/documents/hf ben-xvi enc_20090629 caritas-in-veritate.html

${ }^{31} \mathrm{MM}, \S 69$.

${ }^{32} \mathrm{GS}, \S 69$.

${ }^{33} \mathrm{QA}, \S 67$.
} 
s'accumule aux mains des capitalistes soit réduite à une plus équitable mesure et qu'il s'en répande une suffisante abondance parmi les ouvriers, non certes pour que ceux-ci relâchent leur labeur - l'homme est fait pour travailler comme l'oiseau pour voler, - mais pour qu'ils accroissent par l'épargne un patrimoine qui, sagement administré, les mettra à même de faire face plus aisément et plus sûrement à leurs charges de famille $»^{34}$. L'accession à la propriété ne concerne pas d'abord explicitement le capital de l'entreprise comme cela est indiqué dans l'encyclique Rerum Novarum. Cette solution est en revanche mentionnée par Pie XI qui suggère de combiner droit du travail et droit des sociétés pour permettre l'accession des travailleurs à la propriété du capital de leur entreprise : «Nous estimons cependant plus approprié aux conditions présentes de la vie sociale de tempérer quelque peu, dans la mesure du possible, le contrat de travail par des éléments empruntés au contrat de société. C'est ce que l'on a déjà commencé à faire sous des formes variées, non sans profit sensible pour les travailleurs, et pour les possesseurs du capital. Ainsi les ouvriers et employés ont été appelés à participer en quelque manière à la propriété de l'entreprise, à sa gestion ou aux profits qu'elle apporte $»^{35}$. On constate que la propriété du capital, la participation à la gestion et aux profits ne sont pas ici dissociées par le pape. Jean XXIII insistera encore sur ce point en précisant que plus encore qu'au temps de son prédécesseur, «il est aujourd'hui extrêmement souhaitable que les travailleurs arrivent progressivement à participer, de la façon qui paraîtra convenir le mieux, à la propriété de leur entreprise $»^{36}$. Si cet aspect n'est pas développé par l'initiateur de Vatican II, il révèle une vision intéressante qui n'a pas été développée ensuite à notre connaissance. Le titre des paragraphes 75 à 77 est en effet Créance des travailleurs sur l'autofinancement suggérant que les travailleurs ont des droits sur les flux de trésorerie

\footnotetext{
${ }^{34} \mathrm{QA}, \S 68$.

35 QA, §72.

${ }^{36} \mathrm{MM}, \S 77$.
} 
d'exploitation de leur entreprise après rémunération du capital par les dividendes ${ }^{37}$. Ce sera ensuite Jean-Paul II dans l'encyclique Laborem Exercens (paragraphes 14 à 16) qui fera le plus référence à la participation dans l'entreprise. Il évoque les «propositions concernant la copropriété des moyens de travail, la participation des travailleurs à la gestion et/ou aux profits des entreprises, ce que l'on nomme l'actionnariat ouvrier, etc. $\|^{38}$, aux 4 multiples activités, avec la contribution notable des chrétiens, d'où ont résulté la fondation de coopératives de production, de consommation et de crédit, la promotion de l'instruction populaire et de la formation professionnelle, l'expérimentation de diverses formes de participation à la vie de l'entreprise et, en général, de la société ${ }^{39}$. Jean-Paul II insiste également sur le désir de l'homme qui travaille d' «apparaître comme co-responsable et coartisan au poste de travail qu'il occupe $»^{40}$ et « non seulement de recevoir la rémunération qui lui est due pour son travail, mais aussi qu'on prenne en considération, dans le processus même de production, la possibilité pour lui d'avoir conscience que, même s'il travaille dans une propriété collective, il travaille en même temps «à son compte $» »^{41}$. Si le travailleur ne pouvait pas avoir conscience qu'il travaille «à son compte», le pape avertit des conséquences : «Dans le cas contraire, il s’ensuit nécessairement dans tout le processus économique des dommages incalculables, dommages qui ne sont pas seulement économiques mais qui atteignent avant tout l'homme $»^{42}$.

Pour l'encyclique, l'association à la propriété du capital n'est pas dissociée de la participation aux décisions qui est plutôt envisagée comme sa conséquence. La propriété du capital doit ainsi «donner vie à une série de corps intermédiaires à finalités économiques, sociales et culturelles : ces corps jouiraient d'une autonomie effective vis-à-vis des pouvoirs publics ; ils

\footnotetext{
${ }^{37}$ Nous considérons en effet que l'autofinancement évoqué par le paragraphe 75 de Mater et Magistra n'est autre que la capacité d'autofinancement diminuée de la distribution des dividendes.

${ }^{38} \mathrm{LE}, \S 14$.

${ }^{39} \mathrm{LE}, \$ 16$.

${ }^{40} \mathrm{LE}, \S 15$.

${ }^{41} \mathrm{LE}, \S 15$.

${ }^{42} \mathrm{LE}, \S 15$.
} 
poursuivraient leurs objectifs spécifiques en entretenant entre eux des rapports de loyale collaboration et en se soumettant aux exigences du bien commun, ils revêtiraient la forme et la substance d'une communauté vivante $»^{43}$.

\section{La participation pour la prospérité}

La participation n'est pas uniquement une recommandation morale de l'Eglise. Les encycliques considèrent que la participation permet d'améliorer l'efficacité économique. Léon XIII évoque l'amélioration de la productivité résultant de la participation à la propriété des moyens de production. Ce faisant, il anticipe un argument des théories économiques contemporaines justifiant le partage des profits : «En outre, la terre produira toute chose en plus grande abondance. Car l'homme est ainsi fait, que la pensée de travailler sur un fonds qui est à lui redouble son ardeur et son application. Il en vient même jusqu'à mettre tout son cœur dans une terre qu'il a cultivée lui-même, qui lui promet à lui et aux siens, non seulement le strict nécessaire, mais encore une certaine aisance. Tous voient sans peine les heureux effets de ce redoublement d'activité sur la fécondité de la terre et sur la richesse du pays ${ }^{44}$. Un siècle plus tard, s'ajoute à cet argument celui de la transformation progressive de l'économie vers une économie fondée sur les connaissances et donc sur l'homme. Pour la commémoration de centenaire de Rerum Novarum, Jean-Paul II identifie la principale source de la prospérité des sociétés contemporaines : le travail humain. « $\mathrm{Si}$, autrefois, le facteur décisif de la production était la terre, et si, plus tard, c'était le capital, compris comme l'ensemble des machines et des instruments de production, aujourd'hui le facteur décisif est de plus en plus l'homme lui-même, c'est-à-dire sa capacité de connaissance qui apparaît dans

\footnotetext{
${ }^{43} \mathrm{LE}, \S 14$.

${ }^{44} \mathrm{RN}, \S 35$.
} 
le savoir scientifique, sa capacité d'organisation solidaire et sa capacité de saisir et de satisfaire les besoins des autres $»^{45}$.

Les enseignements de l'Eglise en matière de participation ont fait la preuve de leurs effets bénéfiques. En particulier, l'Eglise ne dissocie par les différentes formes de participation et les recherches montrent que cette préconisation peut produire les meilleurs effets en termes de performance ${ }^{46}$. En France, ces travaux montrent que l'association des formes de participation financière et non financière produisent les meilleurs effets pour la performance sociale ${ }^{47}$. Pendant la première décennie des années 2000, les plus grandes entreprises françaises cotées enregistrent de meilleures performances économiques et financières lorsqu'elles favorisent l'actionnariat salariét ${ }^{48}$. La codétermination allemande - l'obligation pour les conseils d'administration d'attribuer entre un tiers et la moitié des sièges aux salariés - apporte une connaissance opérationnelle de première main à la prise de décision ${ }^{49}$. Outre Atlantique, les preuves sont les plus nombreuses. Les travaux de Douglas Kruse et Joseph Blasi le démontrent depuis les années 90 en particulier pour l'actionnariat salarié ${ }^{50}$. Ces auteurs identifient le partage des profits ou shared capitalism comme une des principales causes du succès économique de la Silicon Valley ${ }^{51}$ et des Etats-Unis depuis le XIX ${ }^{\text {ème }}$ siècle $^{52}$. Douglas

\footnotetext{
${ }^{45} \mathrm{CA}, \S 32$.

${ }^{46}$ Nous avons par ailleurs exposé cet argument de la complémentarité des formes de participation notamment défendue par l'économiste François Perroux (Aubert, N. (2013). Une théorie de la participation fondée sur le don: l'apport de François Perroux. Economies et sociétés, 22(1), 63-92.)

${ }^{47}$ Benhamou, S., \& Diaye, M.-A. (2011). Participation des salariés et performance sociale, quels liens ? Quels enjeux? Document de travail. Paris: Centre d'analyse stratégique.

48 Ginglinger, E., Megginson, W., \& Waxin, T. (2011). Employee ownership, board representation, and corporate financial policies. Journal of Corporate Finance, 17(4), 868-887.

Guedri, Z., \& Hollandts, X. (2008). Beyond Dichotomy: The Curvilinear Impact of Employee Ownership on Firm Performance. Corporate Governance: An International Review, 16(5), 460-474.

49 Fauver, L., \& Fuerst, M. E. (2006). Does good corporate governance include employee representation? Evidence from German corporate boards. Journal of Financial Economics, 82(3), 673-710.

${ }^{50}$ Kruse, D., Blasi, J., \& Freeman, R. (2012). Does linking worker pay to firm performance help the best firms to do even better?, 17745.

Kruse, D., Freeman, R. B., \& Blasi, J. R. (2010). Shared capitalism at work : employee ownership, profit and gain sharing, and broad-based stock options. National Bureau of Economic Research conference report. Chicago: The University of Chicago Press.

${ }^{51}$ Blasi, J. R., Kruse, D., \& Bernstein, A. (2003). In the company of owners : the truth about stock options (and why every employee should have them). New York: Basic Books.

${ }^{52}$ Blasi, J. R., Freeman, R. B., \& Kruse, D. (2013). The citizen's share : putting ownership back into democracy.
} 
Kruse a été senior economist au Council of Economic Advisors de la Maison Blanche de 2013 à 2014. 